\title{
Inflammatory Biomarkers Levels in T2DM Emirati Patients with Diabetic Neuropathy
}

\author{
Bashair M Mussa (D) \\ Ankita Srivastava ${ }^{2}$ \\ Abeer Al-Habshi iD ${ }^{2}$ \\ Abdul Khader Mohammed (iD ${ }^{2}$ \\ Rabih Halwani (iD) 2,3 \\ Salah Abusnana $\mathbb{D D}^{3,4}$ \\ 'Basic Medical Science Department, \\ College of Medicine, University of \\ Sharjah, Sharjah, United Arab Emirates; \\ ${ }^{2}$ Sharjah Institute for Medical Research, \\ University of Sharjah, Sharjah, United \\ Arab Emirates; ${ }^{3}$ Clinical Science \\ Department, College of Medicine, \\ University of Sharjah, Sharjah, United \\ Arab Emirates; ${ }^{4}$ Diabetes and \\ Endocrinology Department, University \\ Hospital Sharjah, Sharjah, United Arab \\ Emirates
}

Introduction: Previous studies have suggested the involvement of chronic low-grade inflammation in the pathogenesis of diabetic neuropathy (DNP). However, none of these studies have examined the levels of monocyte chemoattractant protein-1 (MCP-1) in type 2 diabetes mellitus (T2DM) patients with confirmed diagnosis of neuropathy. Therefore, the present study aims to investigate the levels of MCP-1 along with IL-6, IL-8 and TGF- $\beta$ in patients with T2DM and confirmed neuropathy and identify correlations, if any, between MCP-1 and other parameters.

Methods: A single center cross-sectional clinical study was conducted at University Hospital Sharjah (UHS) and University of Sharjah. One hundred and two patients with T2DM were recruited from diabetes clinics at UHS and were stratified into different groups based on diagnosis of DNP and other parameters. Several analyses were conducted to evaluate and compare the levels of MCP-1, IL-6, IL-8, and TGF- $\beta$ across these groups of patients and identify correlations, if any, between MCP-1 and other variables.

Results: A significant increase was found in the levels of MCP-1 in T2DM patients with DNP compared to the patients without DNP ( $p=0.002, p-a d j=0.007)$. Further analysis has shown that levels of IL- $8(\mathrm{p}=0.008)$ and TGF- $\beta(\mathrm{p}=0.06)$ were increased and decreased, respectively, in patients with DNP compared to patients without DNP. Moreover, strong correlations were found between MCP-1, IL- 8 and TGF- $\beta$ levels.

Conclusion: The key finding of the present study is the significant elevation in levels of MCP-1 in T2DM patients with DNP compared to the patients without DNP and IL-8 and TGF- $\beta$ were strong predictors of MCP-1 increased levels.

Keywords: MCP-1, diabetic neuropathy, type 2 diabetes mellitus, inflammatory biomarkers, Emirati population

\section{Introduction}

Diabetes Mellitus (DM) is one of the most common chronic disorders worldwide and reports from the Diabetes International Foundation have revealed that five of the gulf countries are among the top ten countries for prevalence of DM with an estimated increase of $93.9 \%$ between 2010 and $2030 .{ }^{1}$ It is noteworthy that the increase in the prevalence of DM is strongly associated with multiple prevalent risk factors and complications including obesity, hypertension and diabetic neuropathy (DNP). ${ }^{2-5}$ The latter is considered as the most common complication of Type 2 DM (T2DM) which is associated with high rates of morbidity and mortality in addition to the economic burden which involves an estimated cost of 13 billion dollars. ${ }^{6}$ Moreover, DNP is the leading cause of severe ulcerations and lower extremity amputations and annually about 80,000 amputations are performed in patients with
Correspondence: Bashair M Mussa Basic Medical Science Department, College of Medicine, University of Sharjah, P.O. Box: 27272, Sharjah, United Arab Emirates

Tel $+97 \mid-65057220$

Fax +97I-6558579

Email bmussa@sharjah.ac.ae 
DM. ${ }^{7}$ More than twenty years ago, researchers found that about $30 \%$ to $50 \%$ of the patients with DM develop neuropathy during their lifetime. ${ }^{8}$

Classically, it was believed that hyperglycemia is the main cause of DNP and excess glucose can interrupt several metabolic pathways that eventually lead to inflammatory injury of the nerves. ${ }^{9}$ Therefore, the guidelines of DNP treatment were mainly based on glycemic control which was significantly effective in Type 1 DM (T1DM). However, the therapeutic outcomes in patients with T2DM were much less pronounced suggesting other simultaneously contributing factors for development of DNP in patients with T2DM. ${ }^{10}$

In recent years, an evidence has emerged to suggest that chronic low-grade inflammation is associated with development of T2DM and its neurodegenerative complications. ${ }^{11}$ Clinical studies have demonstrated the involvement of inflammatory cytokines in the pathogenesis of DNP and increased cytokine concentration has been reported in T2DM patients with large nerves damage. ${ }^{12}$ In addition, elevated levels of various biomarkers have been reported in T2DM previously and this include serum sialic acid, $\alpha-1$ acid glycoprotein, C-reactive protein (CRP), serum amyloid A and cortisol. Most importantly, higher concentrations of major cytokine mediators, interleukin-6 (IL-6) and tumour necrosis factor alpha (TNFa), have also been detected in serum of T2DM patients compared to healthy subjects. ${ }^{13}$ In addition, more inflammatory biomarkers such as interleukin-8 (IL-8) and transforming growth Factor-Beta (TGF-b) were studied in T2DM; and these biomarkers were found to increase at the onset of T2DM and to remain elevated throughout the duration of the disease. ${ }^{13}$ This further supports the involvement of inflammatory agents in the pathogenesis and the complications associated with T2DM. ${ }^{14,15}$ Interestingly, these observations have been reported across different ethnic groups including Mexican Americans. ${ }^{14,15}$ It is noteworthy that none of these aspects have been investigated in Middle Eastern populations, in particular, Emirati populations (United Arab Emirates, UAE Citizens).

During the last decade, an exceptional attention has been paid to the involvement of monocyte chemoattractant protein-1 (MCP-1) in the pathogenesis of DM and obesity. ${ }^{16}$ MCP-1 is the first discovered chemokine which regulates migration and infiltration of monocytes/ macrophages in response to inflammation. ${ }^{17}$ High circulating levels of MCP-1 have been found in patients with T2DM and significant correlations have been documented between MCP-1 G-2518 polymorphism, insulin resistance and obesity. Interestingly, this varied among different populations including German Caucasians, Turkish, Chinese, Japanese, Indians and Koreans. ${ }^{12,18-21}$ In addition, further investigation has revealed that MCP-1 is associated with a wide range of T2DM complications which include cardiovascular diseases, nephropathy and retinopathy. ${ }^{22-24}$ However, none of these studies have examined the levels of MCP-1 in T2DM patients with confirmed diagnosis of neuropathy. Therefore, the present study aims to investigate the levels of MCP-1 along with IL-6, IL-8 and TGF- $\beta$ in Emirati patients with T2DM and confirmed neuropathy.

\section{Methods}

\section{Study Population and Design}

The present cross-sectional study was conducted at the University Hospital Sharjah (UHS) and Sharjah Institute for Medical Research (University of Sharjah, UOS). The study was approved by the ethics committee of UHS (UHS 10032019) and UOS (REC-19-01-21-02) and conducted in accordance with the Declaration of Helsinki. All participants were asked to sign an informed-consent form written in their native language. This form had been approved by the ethics committee prior to the recruitment process.

One hundred and two Emirati patients with T2DM were recruited from diabetes clinics in UHS. The inclusion criteria were Emirati citizens aged between 18 and 70 years with T2DM for more than five years. The study was designed to assess the level of different inflammatory markers in different groups of patients with and without DNP. Diagnosis of neuropathy was confirmed by the consultants at UHS which was documented in the electronic medical record data base. The following guidelines were used for diagnosis of neuropathy: (i) all patients were assessed for distal symmetric polyneuropathy starting at diagnosis of T2DM, (ii) the assessment included a careful history and either temperature or pinprick sensation (small-fiber function) and vibration sensation using a 128$\mathrm{Hz}$ tuning fork (large-fiber function) and (iii) patients had an annual $10-\mathrm{g}$ monofilament testing to assess for feet at risk for ulceration and amputation. Finally, electrophysiological testing or referral to a neurologist was rarely needed for screening, except in situations where the clinical features are atypical, the diagnosis is unclear, or a different etiology is suspected. Atypical features 
included motor greater than sensory neuropathy, rapid onset, or asymmetrical presentation.

Given that the main objective of the present study is to examine levels of inflammatory markers in correlation with neuropathy in T2DM, patients with T2DM, BMI < $30 \mathrm{Kg} / \mathrm{m}^{2}$ and without DNP were considered as the control group. The following patients were excluded from this study: T1DM patients, patients with DM duration less than 5 years, aged more than 70 years and those who were acutely ill.

\section{Data and Blood Samples Collection}

Demographic and clinical data for all participants was collected from the UHS electronic medical records system including age, gender, nationality and anthropometric measurements, height $(\mathrm{cm})$, weight $(\mathrm{kgs})$ and BMI $\left(\mathrm{kg} / \mathrm{m}^{2}\right)$. In addition, vital signs were also measured and recorded including systolic (SBP) and diastolic blood pressure (DBP) and heart rate.

About $5 \mathrm{~mL}$ of blood sample was collected from each subject at UHS once and the following laboratory tests were conducted: hemoglobin A1c (HbAlC, \%), fasting blood glucose $(\mathrm{mmol} / \mathrm{L})$, total cholesterol $(\mathrm{mmol} / \mathrm{L})$, triglycerides $(\mathrm{mmol} / \mathrm{L})$, high-density lipoprotein (HDL, $\mathrm{mmol} / \mathrm{L}$ ) and low-density lipoprotein (LDL, $\mathrm{mmol} / \mathrm{L}$ ).

\section{Evaluation of Serum Levels of Inflammatory Biomarkers}

After the whole blood was collected, it was left undisturbed for about 15-30 minutes at room temperature to allow clotting. To obtain serum, clotted blood samples were centrifuged at $2000 \mathrm{RPM}$ for 10 minutes at $4^{\circ} \mathrm{C}$. The serum then was used to detect the levels of the following inflammatory biomarkers: IL-6, IL-8, TGF- $\beta$ and MCP-1 using human Enzyme-Linked Immunosorbent Assay (ELISA) kits (Elabscience, Guangzhou, China) following the manufacturer's protocol.

\section{Statistical Analysis}

Data analysis was performed using the IBM SPSS 26. Data were presented as a mean \pm standard deviation. All non-normal variables were normalized by $\log$ or square root transformation prior to parametric testing. The means between groups were compared using Independent Student's $T$-test. Pearson correlation and linear regression analysis was performed to assess associations between variables of interest. Partial correlation was performed with MCP-1 as a dependent variable after adjusting for potential confounders such as age, gender and BMI. In all tests, $\mathrm{P}$ values less than 0.05 were considered as statistically significant.

\section{Results}

\section{Levels of Inflammatory Biomarkers in T2DM Patients with and without DNP}

Obesity was observed in $46 \%$ of the studied population with T2DM duration average of 15 years and the most common type of neuropathy in the studied population was polyneuropathy. The clinical and anthropometric characteristics of patients without DNP versus those with confirmed diagnosis of DNP are mentioned in Table 1. The total number of patients without DNP was 53 (males: 27; females: 26) whereas the total number of patients with confirmed diagnosis of DNP was 47 (males: 14; females: 33). The minimal age of inclusion was 18 years however other criteria included T2DM for more than five years led to the finding that the mean age for both groups was $60.3 \pm 8.0$ and $60.9 \pm 8.6$ years, respectively, and had no significant difference among these groups $(p=0.70)$. BMI for the patients with DNP was significantly higher $\left(33.5 \pm 4.7 \mathrm{~kg} / \mathrm{m}^{2}\right)$ than the patients without DNP $\left(31.0 \pm 6.1 \mathrm{~kg} / \mathrm{m}^{2}\right)(p=0.03)$ however, both groups are considered obese. Lipid profile including total cholesterol, HDL, LDL, and triglycerides was similar for both groups whereas $\mathrm{HbA1c}$ percentage for DNP patients was slightly higher $(7.9 \pm 1.2)$ than the patients without DNP $(7.6 \pm 1.2)$ however, there was no statistical significance (Table 1).

Evaluation of serum inflammatory biomarkers levels between patients without and with DNP showed that levels of IL-6 did not differ across the two groups prior to or after adjustment of gender, age, BMI and HbA1c (Table 1). On the other hand, TGF- $\beta$ decreased significantly after adjustment of gender, age, BMI and HbAlc $(p=0.03)$. Patients with DNP had significantly higher levels of IL-8 compared to patients without DNP ( $p=0.008)$ and this was a persistent observation after adjusting covariates such as age, gender, BMI and $\mathrm{HbA1c}$ (Table 1). Similarly, patients with confirmed diagnosis of DNP showed significantly higher levels of serum MCP-1 as compared to patients without $\mathrm{DNP}(p=0.002)$ and more importantly the significance persisted after controlling for the covariates (Table 1). 
Table I Anthropometric and Clinical Characteristics and Levels of Inflammatory Biomarkers of Patients without DNP versus Patients with DNP

\begin{tabular}{|c|c|c|c|c|}
\hline Parameters & Without DNP & With DNP & P-value & P-adj \\
\hline$N(M / F)$ & $53(27 / 26)$ & $47(14 / 33)$ & - & - \\
\hline Age (Years) & $60.3 \pm 8.0$ & $60.9 \pm 8.6$ & 0.70 & - \\
\hline BMI $\left(\mathrm{kg} / \mathrm{m}^{2}\right)$ & $31.0 \pm 6.1$ & $33.5 \pm 4.7$ & 0.03 & - \\
\hline SBP $(\mathrm{mmHg})$ & $131.4 \pm 16.5$ & $126.4 \pm 13.8$ & 0.13 & 0.21 \\
\hline $\mathrm{DBP}(\mathrm{mmHg})$ & $78.5 \pm 7.6$ & $77.6 \pm 6.7$ & 0.52 & 0.50 \\
\hline Pulse & $80.0 \pm 10.1$ & $82.3 \pm 10.8$ & 0.30 & 0.25 \\
\hline Glucose $(\mathrm{mmol} / \mathrm{L})$ & $7.9 \pm 2.6$ & $7.6 \pm 2.5$ & 0.59 & 0.62 \\
\hline HbAlc (\%) & $7.6 \pm 1.4$ & $7.9 \pm 1.2$ & 0.28 & - \\
\hline Total Cholesterol (mmol/L) & $4.2 \pm 1.2$ & $4.4 \pm 1.2$ & 0.34 & 0.74 \\
\hline HDL-Cholesterol (mmol/L) & $1.17 \pm 0.30$ & $1.23 \pm 0.27$ & 0.39 & 0.98 \\
\hline LDL-Cholesterol (mmol/L) & $2.41 \pm 1.02$ & $2.58 \pm 1.04$ & 0.46 & 0.76 \\
\hline Triglycerides (mmol/L) & $1.52 \pm 0.77$ & $1.73 \pm 1.11$ & 0.31 & 0.54 \\
\hline IL-6 (pg/mL) & $13.6 \pm 17.4$ & $11.1 \pm 12.1$ & 0.94 & 0.92 \\
\hline $\mathrm{IL-8}(\mathrm{pg} / \mathrm{mL})^{\#}$ & $39.7 \pm 16.4$ & $60.5 \pm 30.7$ & 0.008 & 0.02 \\
\hline TGF- $\beta(p g / m L){ }^{\#}$ & $82.4 \pm 77.1$ & $56.7 \pm 28.8$ & 0.06 & 0.03 \\
\hline MCP-I $(\mathrm{pg} / \mathrm{mL})^{\#}$ & $52.8 \pm 37.4$ & $83.3 \pm 63.5$ & 0.002 & 0.007 \\
\hline
\end{tabular}

Notes: Data were presented as mean \pm standard deviation for normal continuous variables; ${ }^{\#}$ Denotes continuous variables with non-Gaussian distribution and were log or square root transformed prior to analysis. $P$ values less than 0.05 were considered as statistically significant. $P$ adj indicates the $P$ values after adjusting for covariates including age, gender, BMI and $\mathrm{HbAlc}$. The bold text highlights the significant differences between studied groups ( $P$ values less than 0.05$)$.

Levels of Inflammatory Biomarkers in Non-Obese Patients without DNP versus Obese Patients with DNP

To further investigate the inflammatory markers in obesity associated with DNP, we conducted a comparison between non-obese patients without DNP and obese patients with DNP
(Table 2). The analysis of clinical and anthropometric characteristics of these two groups have shown that the total number of patients in the first group was 30 (males: 19; females: 11) whereas the total number of patients in the second group was 47 (males: 14; females: 33). The mean age for both groups was $60.1 \pm 7.3$ and $60.9 \pm 8.6$ years,

Table 3 Anthropometric and Clinical Characteristics and Levels of Inflammatory Biomarkers in Obese and Non-obese Patients with DPN

\begin{tabular}{|c|c|c|c|c|}
\hline Parameters & DPN Obese & DPN Non-Obese & P-value & P-adj \\
\hline$N(M / F)$ & $42(13 / 29)$ & $5(1 / 4)$ & - & - \\
\hline Age (Years) & $60.9 \pm 8.8$ & $60.8 \pm 7.0$ & 0.98 & - \\
\hline BMI (kg/m2) & $34.4 \pm 4.2$ & $26.9 \pm 1.8$ & $<0.0001$ & $<0.0001$ \\
\hline $\mathrm{SBP}(\mathrm{mmHg})$ & $127.1 \pm 13.8$ & $117.3 \pm 10.9$ & 0.24 & 0.23 \\
\hline $\mathrm{DBP}(\mathrm{mmHg})$ & $77.9 \pm 6.7$ & $73.3 \pm 5.0$ & 0.26 & 0.28 \\
\hline Pulse & $82.6 \pm 11.1$ & $79.0 \pm 4.6$ & 0.58 & 0.66 \\
\hline Glucose (mmol/L) & $7.65 \pm 2.6$ & $6.96 \pm 1.2$ & 0.66 & 0.98 \\
\hline HbAlc (\%) & $7.95 \pm 1.2$ & $7.66 \pm 1.0$ & 0.60 & - \\
\hline Total Cholesterol (mmol/L) & $4.5 \pm 1.2$ & $4.1 \pm 1.1$ & 0.52 & 0.52 \\
\hline HDL-Cholesterol (mmol/L) & $1.22 \pm 0.27$ & $1.29 \pm 0.26$ & 0.56 & 0.63 \\
\hline LDL-Cholesterol (mmol/L) & $2.60 \pm 0.97$ & $2.49 \pm 1.19$ & 0.85 & 0.86 \\
\hline Triglycerides (mmol/L) & $1.83 \pm 1.11$ & $0.96 \pm 0.56$ & 0.09 & 0.12 \\
\hline $\mathrm{IL}-6(\mathrm{pg} / \mathrm{mL})^{\#}$ & $10.9 \pm 12.4$ & $13.4 \pm 8.70$ & 0.48 & 0.46 \\
\hline IL-8 (pg/mL) & $60.5 \pm 30.7$ & - & - & - \\
\hline TGF- $\beta(p g / m L))^{\#}$ & $57.9 \pm 30.1$ & $47.4 \pm 12.8$ & 0.34 & 0.25 \\
\hline MCP-I $(\mathrm{pg} / \mathrm{mL})^{\#}$ & $83.7 \pm 64.6$ & $80.5 \pm 60.9$ & 0.79 & 0.81 \\
\hline
\end{tabular}

Notes: Data were presented as mean \pm standard deviation for normal continuous variables; ${ }^{\#}$ Denotes continuous variables with non-Gaussian distribution and were log or square root transformed prior to analysis. $P$ values less than 0.05 were considered as statistically significant. $P$ adj indicates the $P$ values after adjusting for covariates such as age, gender and $\mathrm{HbAlc}$. 
respectively, with no significant difference between the two groups ( $p=0.68$ ). BMI for non-obese without DNP group was $26.8 \pm 2.3 \mathrm{~kg} / \mathrm{m}^{2}$ which was significantly lower than the obese group $\left(33.6 \pm 4.7 \mathrm{~kg} / \mathrm{m}^{2}\right)(p=<0.0001)$. No difference was noted between the lipid profiles, HbAlc levels and the inflammatory biomarker IL-6 in the two groups prior to and after adjustment of the covariates. However, a significant difference was found in the levels of IL-8 (38.4 \pm 19.4 vs $60.5 \pm 30.7$; $p=0.02)$, TGF- $\beta(74.9 \pm 42.1$ vs $56.7 \pm 28.8 ; p=0.01)$ and MCP-1 (48.1 \pm 36.1 vs $83.3 \pm 63.5 ; p=0.001)$ between the two groups. Obese patients with confirmed DNP have higher levels of IL-8 and MCP-1 and lower levels of TGF- $\beta$. Significant difference in the levels of MCP-1 and TGF- $\beta$ persisted after controlling for age, gender and $\mathrm{HbAlc}$ (Table 2).

\section{Levels of Inflammatory Biomarkers in Obese and Non-Obese Patients with DNP}

Although the number of non-obese patients with DNP was small $(n=5,0.04 \%)$, we conducted a comparison between this group of patients and obese patients with DNP $(n=42$, 41\%). As shown in Table 3, there was no significant difference between anthropometric and clinical characteristics nor the levels of inflammatory biomarkers including
MCP-1, TGF- $\beta$ and IL- 6 between the two groups and the same outcomes were noted after adjustment of age, gender and HbA1c. IL-8 was not detectable in nonobese patients with DNP, and this led to limited comparative analysis.

\section{Correlation of MCP-I Levels with Different Clinical and Inflammatory Parameters}

In addition, we performed stepwise linear regression analysis to investigate the association of MCP-1 independent of confounders. Several variables were assessed including age, gender, BMI, SBP, DBP, pulse, glucose, HbA1c, total cholesterol, HDL-cholesterol, LDL-cholesterol, triglycerides, $\log$ IL-6, log IL-8, log TGF- $\beta$. As shown in Table 4, only IL- 8 and TGF- $\beta$ were significant predictors of MCP1 , providing combined prediction of $47 \%$ (adjusted $\mathrm{R}^{2}$ $=0.47, \mathrm{p}=0.04)$ of the variance perceived in MCP-1 levels.

\section{Analysis of the Anti-Inflammatory Medications and T2DM Complications}

We investigated the prescribed anti-inflammatory medications that were used by the studied group of patients, and it was found that $31 \%$ of the patients used Diclofenac and $27 \%$ of the patients used Aspirin whereas a smaller number of patients used Celecoxib (12\%).

Table 2 Anthropometric and Clinical Characteristics and the Levels of Inflammatory Biomarkers in Non-Obese Patient without DNP versus Obese Patients with DNP

\begin{tabular}{|c|c|c|c|c|}
\hline Parameters & Non-Obese without DNP & Obese with DNP & P-value & P-adj \\
\hline$N(M / F)$ & $30(19 / 11)$ & $47(14 / 33)$ & - & - \\
\hline Age (Years) & $60.1 \pm 7.3$ & $60.9 \pm 8.6$ & 0.68 & - \\
\hline BMI $\left(\mathrm{kg} / \mathrm{m}^{2}\right)$ & $26.8 \pm 2.3$ & $33.6 \pm 4.7$ & $<0.0001$ & $<0.0001$ \\
\hline $\mathrm{SBP}(\mathrm{mmHg})$ & $130.3 \pm 15.9$ & $126.4 \pm 13.8$ & 0.28 & 0.50 \\
\hline $\mathrm{DBP}(\mathrm{mmHg})$ & $77.7 \pm 8.5$ & $77.6 \pm 6.8$ & 0.94 & 0.65 \\
\hline Pulse & $79.9 \pm 11.4$ & $82.3 \pm 10.9$ & 0.39 & 0.55 \\
\hline Glucose (mmol/L) & $8.3 \pm 3.1$ & $7.6 \pm 2.5$ & 0.32 & 0.34 \\
\hline HbAlc (\%) & $7.6 \pm 1.3$ & $7.9 \pm 1.2$ & 0.26 & - \\
\hline Total Cholesterol (mmol/L) & $4.1 \pm 1.1$ & $4.4 \pm 1.2$ & 0.24 & 0.33 \\
\hline HDL-Cholesterol (mmol/L) & $1.17 \pm 0.27$ & $1.23 \pm 0.27$ & 0.44 & 0.44 \\
\hline LDL-Cholesterol (mmol/L) & $2.33 \pm 0.91$ & $2.58 \pm 1.04$ & 0.33 & 0.27 \\
\hline Triglycerides (mmol/L) & $1.59 \pm 0.92$ & $1.73 \pm 1.11$ & 0.61 & 0.56 \\
\hline $\mathrm{IL}-6(\mathrm{pg} / \mathrm{mL})^{\#}$ & $10.0 \pm 11.2$ & $11.0 \pm 12.1$ & 0.37 & 0.34 \\
\hline IL-8 (pg/mL) $)^{\#}$ & $38.4 \pm 19.4$ & $60.5 \pm 30.7$ & 0.02 & 0.10 \\
\hline TGF- $\beta(p g / m L))^{\#}$ & $74.9 \pm 42.1$ & $56.7 \pm 28.8$ & 0.01 & 0.01 \\
\hline MCP-I $(\mathrm{pg} / \mathrm{mL})^{\#}$ & $48.1 \pm 36.1$ & $83.3 \pm 63.5$ & 0.001 & 0.01 \\
\hline
\end{tabular}

Notes: Data were presented as mean \pm standard deviation for normal continuous variables; ${ }^{*}$ Denotes continuous variables with non-Gaussian distribution and were log or square root transformed prior to analysis. $P$ values less than 0.05 were considered as statistically significant. $P$ adj indicates the $P$ values after adjusting for covariates including age, gender and HbAlc. The bold text highlights the significant differences between studied groups ( $P$ values less than 0.05$)$. 
Table 4 Stepwise Linear Regression Analysis Using MCP-I as a Dependent Variable

\begin{tabular}{|l|l|l|l|l|}
\hline Model & $\mathbf{R}$ & Adjusted $\mathbf{R}^{2}$ & Standardized $\boldsymbol{\beta}$ & P-value \\
\hline IL-8 & 0.64 & 0.39 & 0.64 & $0.00 \mathrm{I}$ \\
IL-8, TGF- $\beta$ & 0.72 & 0.47 & $0.57,-0.33$ & 0.04 \\
\hline
\end{tabular}

Notes: Independent variables entered in the model: Age, gender, BMI, SBP, DBP, pulse, glucose, HbAIc, total cholesterol, HDL-cholesterol, LDL-cholesterol, triglycerides, log IL-6, log IL-8, log TGF- $\beta$. P values less than 0.05 were considered as statistically significant.

On the other hand, analysis of the complications in the studied group showed that the main dominant complications were hypertension (50\%) and hyperlipidemia (41\%) and to lesser extend vitamin D deficiency which was present in $17 \%$ of the studied population.

\section{Discussion}

Pathogenesis of DNP involves complex biochemical pathways and processes including increased oxidative stress, activation of the polyol and protein kinase $C$ pathways, impaired blood supply to nerves, neuronal apoptosis and low-grade inflammation. ${ }^{12,25}$ It is evident that the latter play a key role in the development of DM complications, in particular, DNP and therefore, exploring the inflammatory biomarkers in patients with T2DM and DNP represents an important goal to better our understanding of the involvement of these markers in the development of DNP.

The present study is the first to investigate the levels of MCP-1 in Emirati patients with T2DM and DNP. One of the key findings of the study is that MCP-1 levels were significantly higher in patients with DNP compared to patients without DNP. In agreement with this finding, previous studies have hypothesized a relationship between MCP-1 and T2DM. ${ }^{16,26}$ It has been found that circulating MCP-1 significantly increased in patients with T2DM and this was more obvious in patients with cardiovascular complications. ${ }^{27}$ In agreement with these findings, the present study reported that $50 \%$ of the studied population have hypertension and $41 \%$ have hyperlipidemia. In addition, molecular studies have shown a strong correlation between the A-2518G polymorphism in the MCP-1 gene, insulin resistance and $\mathrm{T} 2 \mathrm{DM}$ in a large cohort of Caucasians. $^{18}$ Interestingly, an additional MCP-1 rs1024611 polymorphism was studied in 135 patients with diabetes and a strong association between this polymorphism and diabetic foot ulcers was documented. ${ }^{28}$

On the other hand, a recent study was conducted in German population demonstrated higher levels of proinflammatory cytokines such as IL-6, TNF- $\alpha$ and IL-1 receptor antagonist which were positively associated with progression of distal sensorimotor polyneuropathy. ${ }^{29}$ In contrast, low levels of IL-6 were reported in T2DM Emirati population with DNP compared to Caucasians, this finding is supported by previous studies which have revealed a significant variation in IL-6 levels across different races and low levels of IL-6 in Asian population. ${ }^{30}$ Although several studies implicated MCP-1 in the pathogenesis of T2DM complications including diabetic retinopathy and nephropathy, none of these studies examine the role of MCP-1 in DNP and this further emphasizes the novelty of the present findings. ${ }^{31,32}$ In addition, a significant increase was also noted in levels of IL-8 in DNP patients before and after adjustment of gender, age, BMI and HbAlc. This combined increase in levels of MCP-1 and IL-8, which belong to the same inflammatory chemokine family, in patients with DNP indicates that these two agents are involved in the inflammatory process that is associated with DNP. In agreement with these findings, Straczkowski et al have reported increased plasma levels of IL-8 in subjects with impaired glucose tolerance compared to normoglycemic weight-matched individuals. ${ }^{33}$ Interestingly, elevated levels of IL-8 were also observed in other types of neuropathies including optic neuropathy and similar to the present study, no effects on the levels of IL-6 were observed in the patients with optic neuropathy. ${ }^{34}$ It is noteworthy that although anti-inflammatory medications including Diclofenac, Aspirin and Celecoxib were used by $31 \%, 27 \%$ and $12 \%$ of the patients, respectively, the levels of MCP-1 and IL-8 were high, and no correlations were found between antiinflammatory medications and the inflammatory profile of the studied population.

In addition, our current findings have shown a significant decrease in TGF- $\beta$ levels in patients with DNP compared to patients without DNP after adjustment of gender, age, BMI and HbA1c. TGF- $\beta$ is known for its anti-cytokine activity and this provides a reasonable explanation for the inverse relationship between DNP and TGF$\beta{ }^{35}$ Moreover, these findings suggest that low level of TGF- $\beta$ could be a contributing factor in the pathophysiology of DNP however, further studies are required to draw this conclusion. On the other hand, investigation of the other parameters in the present study, showed that no significant differences between patients with and without DNP and this includes the demographic data, vital signs, 
DM control and lipid profile before or after adjustment of gender, age, BMI and $\mathrm{HbA} 1 \mathrm{c}$.

The current investigation of obesity associated with DNP showed that obese patients with DNP have significantly higher levels of IL8 and MCP-1 and low levels of TGF- $\beta$ compared to non-obese patients with no DNP. For further validation of these outcomes related to obesity we conducted a comparison between obese patients with DNP versus non-obese patients with DNP and interestingly no significant differences were found between the levels of inflammatory markers including MCP-1 between the two groups. This finding emphasizes that the significant change that was observed in the levels of MCP-1 are mainly due to DNP in the studied population and not directly related to obesity. However, it is noteworthy that the number of the non-obese patients with DNP was much less compared to the obese patients with DNP and this may influence the validity of the conclusion about the minor role of obesity in the inflammatory process associated with DNP and the relationship between the absence of detectable levels of IL- 8 in non-obese patients with DNP and obesity.

Analysis of the predictors of MCP-1 levels has revealed that IL- 8 and TGF- $\beta$ are the strongest predictors compared to all other variables. This finding was supported by other investigations which showed a strong correlation between chemotactic cytokines, MCP-1 and IL-8 and various physiological conditions including accumulation of adipose tissues, diabetes and atherosclerosis. ${ }^{36}$

In addition, a correlation between levels of MCP-1 and TGF- $\beta$ has been documented previously however, in different inflammatory diseases such nephritis. ${ }^{37}$ The significant and negative correlation between MCP- 1 and TGF- $\beta$ in DNP is considered as a novel finding and no previous reports have claimed this observation.

It is noteworthy that no significant differences were noticed in the levels of IL-6 in any of the comparisons suggesting that in this group of patients this agent does not involve in the inflammatory process that is associated with DNP. In agreement with this finding, a study by Magrinelli et al has demonstrated that IL-6 was only associated with subtype of DNP in which large nerve fibers were damaged, but no relation was established with other types of DNP nor neuropathic pain. ${ }^{38}$

\section{Limitations of the Study}

One of the main limitations of the present study is the small sample size and the recruitment of more participants was a challenge due to the breakout of coronavirus pandemic and the flow of the patients with T2DM was influenced, negatively. In addition, the number of participants in the group of non-obese with DNP was very small and this limited our ability to draw a strong conclusion regarding the analysis of their inflammatory profile, in particular, levels of IL-8 which were not detectable in this group of participants.

\section{Conclusion}

The present study has shown that MCP-1 levels were significantly high in T2DM patients with confirmed DNP after covariate adjustment. In addition, significant differences in the levels of IL- 8 and TGF- $\beta$ were correlated to DNP. TGF- $\beta$ levels were significantly lower in patients with confirmed DNP compared to patients without DNP. Moreover, strong correlations were found between MCP-1 levels and IL-8 and TGF- $\beta$.

We believe that these findings represent a significant step to identify biomarkers for DNP in T2DM patients which helps in screening and stratification of this group of patients for potential therapeutic targets and implementation of intervention strategies. In addition, further studies can provide more in-depth analysis of the correlation between the inflammatory biomarkers, pain score and amputations in T2DM patients with DNP. Moreover, future studies can include comprehensive investigations of the molecular and genetic features of MCP-1 and DNP.

\section{Acknowledgments}

The authors would like to thank all people who involved in the present study for their valuable participation. In addition, the authors would like to thank Ms. Asma Obaideen for her help in verification of the medical records.

\section{Funding}

This study was supported by Collaborative Grant (NO. 1701090120-P) from University of Sharjah, and Al Jalila Foundation (AJF2018082) to Bashair M. Mussa.

\section{Disclosure}

The authors have no conflicts of interest to declare for this work, financial or otherwise.

\section{References}

1. International Diabetes Federation. IDF Diabetes Atlas. 4th ed. Brussels, Belgium; 2009.

2. Alhyas L, McKay A, Balasanthiran A, Majeed A. Prevalence of overweight, obesity, hyperglycaemia, hypertension and dyslipidaemia in the Gulf: systematic review. JRSM Short Rep. 2011;2(7):55. doi:10.1258/shorts.2011.011019 
3. Adler AI, Neil HA, Manley SE, Holman RR, Turner RC. Hyperglycemia and hyperinsulinemia at diagnosis of diabetes and their association with subsequent cardiovascular disease in the United Kingdom prospective diabetes study (UKPDS 47). Am Heart J. 1999;138(5):353-359. doi:10.1016/S0002-8703(99)70035-9

4. Arauz-Pacheco C, Parrott MA, Raskin P; American Diabetes Association. Treatment of hypertension in adults with diabetes. Diabetes Care. 2003;26(Suppl 1):S80-82.

5. Mussa BM, Schuaman M, Jomma Y, Abusnana S. Prevalence of hypertension and obesity in Emirati patients with type 2 diabetes. J Diabetes Metab. 2016;7:1.

6. Gordois A, Scuffham P, Shearer A, Oglesby A, Tobian JA. The health care costs of diabetic peripheral neuropathy in the US. Diabetes Care. 2003;26(6):1790-1795. doi:10.2337/diacare.26.6.1790

7. Margolis DJ, Malay DS, Hoffstad OJ, et al. Incidence of diabetic foot ulcer and lower extremity amputation among medicare beneficiaries, 2006 to 2008: data points \#2. In: Data Points Publication Series. Rockville (MD): Agency for Healthcare Research and Quality (US); 2011.

8. Maser RE, Steenkiste AR, Dorman JS, et al. Epidemiological correlates of diabetic neuropathy. Report from Pittsburgh Epidemiology of Diabetes Complications Study. Diabetes. 1989;38(11):1456-1461. doi:10.2337/diab.38.11.1456

9. Vincent AM, Hinder LM, Pop-Busui R, Feldman EL. Hyperlipidemia: a new therapeutic target for diabetic neuropathy. $J$ Peripher Nerv Syst. 2009;14(4):257-267. doi:10.1111/j.15298027.2009.00237.x

10. Boulton A. Management of diabetic peripheral neuropathy. Clin Diabetes. 2005;23(1):9-15. doi:10.2337/diaclin.23.1.9

11. Jin HY, Park TS. Role of inflammatory biomarkers in diabetic peripheral neuropathy. J Diabetes Investig. 2018;9(5):1016-1018. doi:10.1111/jdi.12794

12. Doupis J, Lyons TE, Wu S, et al. Microvascular reactivity and inflammatory cytokines in painful and painless peripheral diabetic neuropathy. J Clin Endocrinol Metab. 2009;94(6):2157-2163. doi:10.1210/jc.2008-2385

13. Azar ST, Salti I, Zantout MS, Major S. Alterations in plasma transforming growth factor beta in normoalbuminuric type 1 and type 2 diabetic patients. J Clin Endocrinol Metab. 2000;85(12):4680-4682. doi:10.1210/jcem.85.12.7073

14. Pickup JC, Chusney GD, Thomas SM, Burt D. Plasma interleukin-6, tumour necrosis factor alpha and blood cytokine production in type 2 diabetes. Life Sci. 2000;67(3):291-300. doi:10.1016/S0024-3205(00) 00622-6

15. Mirza S, Hossain M, Mathews C, et al. Type 2-diabetes is associated with elevated levels of TNF-alpha, IL-6 and adiponectin and low levels of leptin in a population of Mexican Americans: a Cross-Sectional Study. Cytokine. 2012;57(1):136-142. doi:10.1016/ j.cyto.2011.09.029

16. Panee J. Monocyte chemoattractant protein 1 (MCP-1) in obesity and diabetes. Cytokine. 2012;60(1):1-12. doi:10.1016/j.cyto.2012.06.018

17. Deshmane SL, Kremlev S, Amini S, Sawaya BE. Monocyte chemoattractant protein-1 (MCP-1): an overview. J Interferon Cytokine Res. 2009;29(6):313-326. doi:10.1089/jir.2008.0027

18. Simeoni E, Hoffmann MM, Winkelmann BR, et al. Association between the A-2518G polymorphism in the monocyte chemoattractant protein-1 gene and insulin resistance and type 2 diabetes mellitus. Diabetologia. 2004;47(9):1574-1580. doi:10.1007/s00125-004-1494-4

19. Karadeniz M, Erdogan M, Cetinkalp S, Berdeli A, Eroglu Z, Ozgen AG. Monocyte chemoattractant protein-1 (MCP-1) 2518G/A gene polymorphism in Turkish type 2 diabetes patients with nephropathy. Endocrine. 2010;37(3):513-517. doi:10.1007/s12020010-9342-4

20. Kouyama K, Miyake K, Zenibayashi M, et al. Association of serum MCP-1 concentration and MCP-1 polymorphism with insulin resistance in Japanese individuals with obese type 2 diabetes. Kobe J Med Sci. 2008;53(6):345-354.
21. Katakami N, Matsuhisa M, Kaneto H, et al. Monocyte chemoattractant protein-1 (MCP-1) gene polymorphism as a potential risk factor for diabetic retinopathy in Japanese patients with type 2 diabetes. Diabetes Res Clin Pract. 2010;89(1):e9-12. doi:10.1016/j. diabres.2010.04.006

22. Mazurek T, Zhang L, Zalewski A, et al. Human epicardial adipose tissue is a source of inflammatory mediators. Circulation. 2003;108 (20):2460-2466. doi:10.1161/01.CIR.0000099542.57313.C5

23. Capeans C, De Rojas MV, Lojo S, Salorio MS. C-C chemokines in the vitreous of patients with proliferative vitreoretinopathy and proliferative diabetic retinopathy. Retina. 1998;18 (6):546-550.

24. Chow FY, Nikolic-Paterson DJ, Ma FY, Ozols E, Rollins BJ, Tesch GH. Monocyte chemoattractant protein-1-induced tissue inflammation is critical for the development of renal injury but not type 2 diabetes in obese $\mathrm{db} / \mathrm{db}$ mice. Diabetologia. 2007;50 (2):471-480. doi:10.1007/s00125-006-0497-8

25. Mahmoud A, Soliman M, Moustafa A. Evaluation of monocyte chemoattractant protein $1(\mathrm{MCP}-1)$ as a predictor of complications in type 2 diabetes mellitus in Zagazig University Hospital. Egypt $J$ Hosp Med. 2021;83(1):995-1001. doi:10.21608/ejhm.20 21.160038

26. Pop-Busui R, Boulton A, Feldman E, et al. Diabetic neuropathy: a position statement by the American Diabetes Association. Diabetes Care. 2017;40(1):136-154. doi:10.2337/dc16-2042

27. Bláha V, Andrýs C, Smahelová A, et al. Effect of atorvastatin on soluble CD14, CD40 Ligand, sE- and sP-selectins and MCP-1 in patients with type 2 diabetes mellitus: relationship to cholesterol turnover. Pharmacol Res. 2006;54(6):421-428. doi:10.1016/j. phrs.2006.08.005

28. Su N, Zhao N, Wang G, et al. Association of MCP-1 rs1024611 polymorphism with diabetic foot ulcers. Medicine (Baltimore). 2018;97(28):e11232. doi:10.1097/MD.0000000000011232

29. Herder C, Kannenberg JM, Huth C, et al. Proinflammatory cytokines predict the incidence and progression of distal sensorimotor polyneuropathy: KORA F4/FF4 Study. Diabetes Care. 2017;40 (4):569-576. doi:10.2337/dc16-2259

30. Coe CL, Love GD, Karasawa M, et al. Population differences in proinflammatory biology: Japanese have healthier profiles than Americans. Brain Behav Immun. 2011;25(3):494-502. doi:10.1016/ j.bbi.2010.11.013

31. Taghavi Y, Hassanshahi G, Kounis NG, Koniari I, Khorramdelazad H. Monocyte chemoattractant protein-1 (MCP-1/ CCL2) in diabetic retinopathy: latest evidence and clinical considerations. J Cell Commun Signal. 2019;13(4):451-462. doi:10.1007/s12079-018-00500-8

32. Banba N, Nakamura T, Matsumura M, Kuroda H, Hattori Y, Kasai K. Possible relationship of monocyte chemoattractant protein-1 with diabetic nephropathy. Kidney Int. 2000;58(2):684-690. doi:10.1046/ j.1523-1755.2000.00214.x

33. Straczkowski M, Kowalska I, Nikolajuk A, Dzienis-Straczkowska S, Szelachowska M, Kinalska I. Plasma interleukin 8 concentrations in obese subjects with impaired glucose tolerance. Cardiovasc Diabetol. 2003;2(1):5. doi:10.1186/1475-2840-2-5

34. Goldenberg-Cohen N, Kramer M, Bahar I, Monselise Y, Weinberger D. Elevated plasma levels of interleukin 8 in patients with acute anterior ischaemic optic neuropathy. $\mathrm{Br} J$ Ophthalmol. 2004;88(12):1538-1540. doi:10.1136/bjo.2004.046524

35. Roberts AB, Sporn MB. Physiological actions and clinical applications of transforming growth factor-beta (TGF-beta). Growth Factors. 1993;8(1):1-9. doi:10.3109/08977199309029129

36. Kim CS, Park HS, Kawada T, et al. Circulating levels of MCP-1 and IL-8 are elevated in human obese subjects and associated with obesity-related parameters. Int $J$ Obes (Lond). 2006;30(9):1347-1355. Epub 2006 Mar 14. PMID: 16534530 . doi:10.1038/sj.ijo.0803259 
37. Wagrowska-Danilewicz M, Stasikowska O, Danilewicz M Correlative insights into immunoexpression of monocyte chemoattractant protein-1, transforming growth factor beta-1 and CD68+ cells in lupus nephritis. Pol J Pathol. 2005;56(3):115-120. PMID: 16334978 .
38. Magrinelli F, Briani C, Romano M, et al. The association between serum cytokines and damage to large and small nerve fibers in diabetic peripheral neuropathy. J Diabetes Res. 2015;2015:547834. doi:10.1155/2015/547834

Diabetes, Metabolic Syndrome and Obesity: Targets and Therapy

\section{Publish your work in this journal}

Diabetes, Metabolic Syndrome and Obesity: Targets and Therapy is an international, peer-reviewed open-access journal committed to the rapid publication of the latest laboratory and clinical findings in the fields of diabetes, metabolic syndrome and obesity research. Original research, review, case reports, hypothesis formation, expert opinion and commentaries are all considered for publication. The manuscript management system is completely online and includes a very quick and fair peer-review system, which is all easy to use. Visit http://www.dovepress.com/testimonials.php to read real quotes from published authors. 